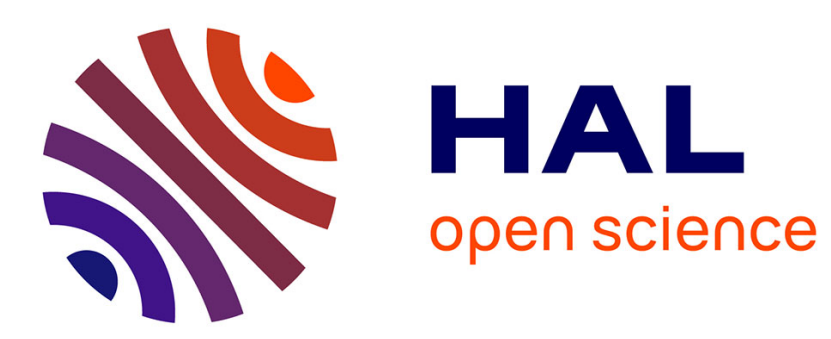

\title{
Local and global instabilities in the wake of a sphere
}

Benoît Pier

\section{To cite this version:}

Benoit Pier. Local and global instabilities in the wake of a sphere. Journal of Fluid Mechanics, 2008, 603, pp.39-61. 10.1017/S0022112008000736 . hal-00206267v2

\section{HAL Id: hal-00206267 \\ https://hal.science/hal-00206267v2}

Submitted on 4 Mar 2009

HAL is a multi-disciplinary open access archive for the deposit and dissemination of scientific research documents, whether they are published or not. The documents may come from teaching and research institutions in France or abroad, or from public or private research centers.
L'archive ouverte pluridisciplinaire HAL, est destinée au dépôt et à la diffusion de documents scientifiques de niveau recherche, publiés ou non, émanant des établissements d'enseignement et de recherche français ou étrangers, des laboratoires publics ou privés. 


\title{
Local and global instabilities in the wake of a sphere
}

\author{
BENOÎT PIER \\ Laboratoire de mécanique des fluides et d'acoustique, \\ École centrale de Lyon, CNRS, Université Claude-Bernard Lyon 1, INSA Lyon, \\ 36 avenue Guy-de-Collongue, 69134 Écully cedex, France
}

(Received 27 July 2007 and in revised form 16 January 2008)

The global dynamics of open shear flows is closely related to the nature of their local instability characteristics, either convective or absolute. The present investigation revisits the wake of a sphere, obtains its global behaviour by direct numerical simulations and derives its local stability features, computed for the underlying basic flow under a quasi-parallel flow assumption. It is shown that both the axisymmetric and the planar symmetric basic flows exhibit domains of local absolute instability in the near-wake region. The largest absolute growth rates occur for instabilities developing on the non-axisymmetric basic wake and conserving its planar symmetry.

\section{Introduction}

The global dynamics of shear flows is known to closely depend on the local instability characteristics, either convective or absolute (Huerre 2000). Convectively unstable systems are sensitive to inflow perturbations and they behave as amplifiers of external noise. In contrast, absolutely unstable systems display non-trivial dynamics without external input, often leading to self-sustained oscillations. Thus, onset of absolute instability is generally associated with drastic changes in the naturally selected global flow features. With this in mind, the present investigation has been undertaken to work out the detailed instability properties prevailing in the wake of a sphere. By analysing the wake structure and its local stability characteristics, it is attempted to establish a link between the naturally selected global flow and the dynamics prevailing locally. A similar line of thought has already been sucessfully implemented for the two-dimensional wake of a cylinder (Pier 2002). This approach is here generalized to the fully three-dimensional flow around a solid sphere.

At moderate Reynolds numbers, the global dynamics of the wake flow around a sphere is now fairly well established, both experimentally and numerically (Roos \& Willmarth 1971; Nakamura 1976; Sakamoto \& Haniu 1995; Johnson \& Patel 1999; Mittal 1999; Ghidersa \& Duček 2000; Tomboulides \& Orszag 2000; Schouveiler \& Provansal 2002; Bouchet, Mebarek \& Duček 2006). The steady axisymmetric wake is stable for $R e<R e_{1} \simeq 212$ and a steady non-axisymmetric flow is observed for $R e_{1}<R e<R e_{2} \simeq 272$. This non-axisymmetric wake displays a symmetry plane and is characterized by two vortex threads aligned with the outer flow. At $R e_{2}$, a Hopf bifurcation leads to periodic vortex shedding. The resulting time-harmonic regime conserves the planar symmetry and the associated Strouhal frequency is in the range 
$0.12<S t<0.18$. At even larger Reynolds numbers, the planar symmetry is lost (Mittal 1999) and further bifurcations lead to a more complex behaviour.

Monkewitz (1988b) investigated the linear stability of an analytic two-parameter family of model axisymmetric, locally parallel and incompressible wake profiles. In that study, the first helical mode was found to display the largest growth rates and to be the only one to become absolutely unstable for velocity profiles approximating those found in the near wake.

Natarajan \& Acrivos (1993) computed the axisymmetric basic flow past a sphere and examined its global linear instability to three-dimensional modal perturbations. The first instability was found at $R e \simeq 210$, associated with a vanishing frequency (regular bifurcation). At $R e \simeq 277.5$, a second mode was found to become unstable via a Hopf bifurcation. While these authors only investigated the stability of the axisymmetric wake, this second critical Reynolds number is remarkably close to $R e_{2} \simeq 272$, corresponding to the transition from a steady non-axisymmetric wake to periodic vortex shedding.

More recently, the role of local absolute instability in self-sustained oscillations developing in three-dimensional axisymmetric flows has been analysed by Sevilla \& Martínez-Bazán (2004) for the wake of a bullet-shaped body, by Gallaire et al. (2006) for spiral vortex breakdown, and by Lesshafft et al. (2006) and Nichols, Schmid \& Riley (2007) for variable-density round jets. These studies have all confirmed the importance of local absolute instability in triggering large-scale global oscillations of these spatially developing flows.

The present investigation has been undertaken in the same spirit and revisits the wake of a sphere in order to establish its complete local absolute instability characteristics. Unlike the above studies, however, not only axisymmetric but also planar symmetric basic wake flows are considered here. Indeed, at $R e_{2}$, onset of vortex shedding occurs via a bifurcation from a planar symmetric basic flow, which therefore requires stability analyses of these non-axisymmetric velocity profiles.

The paper is organized as follows. The governing equations and numerical solution methods for the flow around a sphere are presented in $\$ 2$. The results obtained by direct numerical simulations are given in $\S 3$. This section serves two purposes: first, to validate the numerical code by accurately reproducing the different known flow regimes; second, to obtain the exact time-independent solutions of the Navier-Stokes equations that are used as basic flows for the subsequent stability analyses. In $\S 4$, the local linear stability analyses are carried out for both the axisymmetric and the non-axisymmetric basic flows. Here the local dispersion relations are derived, absolute frequencies are computed and the existence of absolutely unstable domains is established.

\section{Governing equations and numerical solution methods}

The following study is carried out for incompressible flows governed by the NavierStokes equations. The Reynolds number is defined as $R e=U_{\infty} D / v$, where $U_{\infty}$ represents the free-stream velocity, $D$ the sphere diameter and $v$ the kinematic viscosity.

Throughout this investigation, cylindrical coordinates are used with $r, \theta$ and $z(u, v$ and $w$ ) denoting radial, azimuthal and axial coordinates (velocities) respectively. The $z$-axis is aligned with the free-stream velocity and has its origin at the center of the sphere. For future use, a Cartesian $(x, y, z)$-frame is also introduced where the $x$ - and $y$-axes coincide with the directions defined by $\theta=0$ and $\theta=\pi / 2$ respectively. 
Using non-dimensional variables based on $U_{\infty}, D$ and $v$, and denoting the total velocity and pressure fields by $\boldsymbol{u}(r, \theta, z, t)$ and $p(r, \theta, z, t)$ respectively, the governing momentum and continuity equations may be written as

$$
\begin{aligned}
\partial_{t} \boldsymbol{u}+(\boldsymbol{u} \cdot \nabla) \boldsymbol{u}+\nabla p & =\frac{1}{R e} \Delta \boldsymbol{u}+\boldsymbol{f}, \\
\nabla \cdot \boldsymbol{u} & =0,
\end{aligned}
$$

where the different terms are defined in cylindrical coordinates as

$$
\begin{aligned}
\boldsymbol{u} & \equiv\left(\begin{array}{c}
u \\
v \\
w
\end{array}\right), \quad \nabla p \equiv\left(\begin{array}{c}
\partial_{r} p \\
\left(\partial_{\theta} p\right) / r \\
\partial_{z} p
\end{array}\right), \\
(\boldsymbol{u} \cdot \nabla) \boldsymbol{u} & \equiv\left(u \frac{\partial}{\partial r}+\frac{1}{r} v \frac{\partial}{\partial \theta}+w \frac{\partial}{\partial z}\right) \boldsymbol{u}+\frac{1}{r}\left(\begin{array}{c}
-v^{2} \\
u v \\
0
\end{array}\right), \\
\Delta \boldsymbol{u} & \equiv\left(\frac{\partial^{2}}{\partial r^{2}}+\frac{1}{r^{2}} \frac{\partial^{2}}{\partial \theta^{2}}+\frac{\partial^{2}}{\partial z^{2}}\right) \boldsymbol{u}+\frac{1}{r} \frac{\partial \boldsymbol{u}}{\partial r}+\frac{1}{r^{2}}\left(\begin{array}{c}
-u-2 \partial_{\theta} v \\
-v+2 \partial_{\theta} u \\
0
\end{array}\right), \\
\nabla \cdot \boldsymbol{u} & \equiv \frac{\partial u}{\partial r}+\frac{1}{r} u+\frac{1}{r} \frac{\partial v}{\partial \theta}+\frac{\partial w}{\partial z},
\end{aligned}
$$

and $\boldsymbol{f}(r, \theta, z, t)$ is an externally applied volume force to be specified below. The boundary conditions are vanishing velocity fields on the sphere surface and freestream conditions in the far field:

$$
\begin{array}{rlll}
u=0 & \text { for } & r^{2}+z^{2}=1 / 4, \\
u=v=w-1=p=0 & \text { for } \quad r \rightarrow \infty \quad \text { or } \quad z \rightarrow \pm \infty .
\end{array}
$$

\subsection{Immersed boundary method}

For the purpose of fast numerical integration, the above equations are discretized on a Cartesian grid in the $(r, z)$-plane, and the spherical obstacle is treated by an immersed boundary method (for a review of this technique see Mittal \& Iaccarino (2005)). Thus the entire space is assumed to be filled with fluid, and a body force $\boldsymbol{f}_{b}$ is applied inside the region covered by the sphere $\left(r^{2}+z^{2} \leqslant 1 / 4\right)$ so that the fluid is brought to rest there and condition $(2.2 a)$ is satisfied. In the present investigation, several implementations for the force $\boldsymbol{f}_{b}$ have been tested to drive the components of the velocity fields to negligible values. Best results have been obtained when the effect of the sphere is modelled via a visco-elastic restoring force

$$
\boldsymbol{f}_{b}=-\left(\frac{1}{\tau_{v}} \boldsymbol{u}+\frac{1}{\tau_{e}^{2}} \int_{0}^{t} \boldsymbol{u}(\tau) \mathrm{d} \tau\right) \phi\left(\sqrt{r^{2}+z^{2}}\right),
$$

with $\tau_{v}=0.01$ and $\tau_{e}=1$; the shape function is defined as

$$
\phi(\rho) \equiv \frac{1}{2}\left(1+\tanh \frac{0.5-\rho}{\delta}\right)
$$

with $\delta=0.001$.

By implementing this method, there is no need for body-fitted coordinates or explicit boundary conditions on the obstacle surface, thus enabling fast computations 
on a Cartesian $(r, z)$-grid. Another advantage is that the hydrodynamic drag and lift forces acting on the sphere (see \$3.4) are directly obtained by spatial integration of the volume force $f_{b}$ and there is no need to evaluate components of the stress tensor near the surface.

\subsection{Spatial discretization}

All flow quantities are expanded as Fourier modes in the azimuthal direction (indexed by the integer $\beta$ )

$$
\boldsymbol{u}(r, \theta, z, t)=\sum_{\beta} \boldsymbol{u}_{\beta}(r, z, t) \mathrm{e}^{\mathrm{i} \beta \theta} \quad \text { and } \quad p(r, \theta, z, t)=\sum_{\beta} p_{\beta}(r, z, t) \mathrm{e}^{\mathrm{i} \beta \theta},
$$

their complex components satisfying the conditions $u_{-\beta}=u_{\beta}^{\star}, v_{-\beta}=v_{\beta}^{\star}, w_{-\beta}=w_{\beta}^{\star}$ and $p_{-\beta}=p_{\beta}^{\star}$, where $\star$ denotes complex conjugation. However, when considering flow fields which exhibit a symmetry plane containing the $z$-axis, the numerical effort may be reduced: if the flow is symmetric about the $(x, z)$-plane, i.e. about the $(\theta=0)$-plane, it is invariant under the planar symmetry operator $\Pi$ mathematically defined as

$$
\Pi:\left(\begin{array}{c}
u(r, \theta, z, t) \\
v(r, \theta, z, t) \\
w(r, \theta, z, t) \\
p(r, \theta, z, t)
\end{array}\right) \mapsto\left(\begin{array}{r}
u(r,-\theta, z, t) \\
-v(r,-\theta, z, t) \\
w(r,-\theta, z, t) \\
p(r,-\theta, z, t)
\end{array}\right),
$$

and the components $u_{\beta}, w_{\beta}$ and $p_{\beta}$ are then real while $v_{\beta}$ is purely imaginary (and $v_{0}=0$ ). In the numerical implementation, the expansions (2.4) are truncated at a finite number of harmonics $|\beta| \leqslant N_{h}$. For the moderate Reynolds numbers of interest here, it has been found that only a small number of modes $\left(N_{h}=3\right.$ or 4$)$ are required to accurately capture all the flow features, a fact already noticed by Ghidersa \& Duček (2000). Note that analyses of the axisymmetric wake features are simply carried out by setting $N_{h}=0$.

The discretization of the $(r, z)$-plane on a Cartesian grid combines finite differences in the $z$-direction with Chebyshev collocation points in the $r$-direction (Canuto, Hussaini \& Quarteroni 1988; Boyd 2001).

An axial mesh of $n_{z}=n_{u}+n_{o}+n_{d}$ points is constructed with $n_{o}$ equispaced grid points separated by $\delta_{z}$ and clustered around $z=0$, and $n_{u}\left(n_{d}\right)$ elements in the upstream (downstream) regions uniformly stretched according to a stretching factor of $\kappa_{u}\left(\kappa_{d}\right)$. All the results shown in this paper have been obtained with $n_{u}=142$, $n_{o}=101, n_{d}=335, \delta_{z}=0.01, \kappa_{u}=\kappa_{d}=1.02$. The total streamwise extent of the domain is then $-8.5<z<387$.

The radial discretization of the $r$-axis on $n_{r}$ collocation points is obtained by mapping the Chebyshev points $-1 \leqslant \xi_{i} \equiv-\cos \left[i \pi /\left(n_{r}+1\right)\right] \leqslant+1$ for $i=0, \ldots, n_{r}+1$ onto the entire radial axis $-\infty \leqslant r_{i} \leqslant+\infty$ through the algebraic transformation $r \sqrt{2} / \ell_{r}=\xi /\left(1-\xi^{2}\right)$. The parameter $\ell_{r}$ governs the distribution of collocation points over the $r$-axis: half of the points are located in the interval $-\ell_{r}<r<\ell_{r}$. Using the vanishing boundary conditions $(2.2 b)$ at $r= \pm \infty$, the computation may be restricted to the interior collocation points associated with $1 \leqslant i \leqslant n_{r}$. Moreover, taking advantage of radial parity properties of the Fourier components (2.4), only positive $r$-values need to be taken into account: the components $u_{\beta+1}, v_{\beta+1}, w_{\beta}$ and $p_{\beta}$ are symmetric (antisymmetric) in $r$ for $\beta$ even (odd). The use of the appropriate (anti)symmetric operators when computing radial derivatives then also resolves the apparent singularity at $r=0$, due to the formulation of the governing equations in 
polar coordinates (Boyd 2001). Throughout, $\ell_{r}=1$ has been used. Different resolution tests have shown that reasonable but approximate results are obtained with $n_{r}=80$, and that a high accuracy is achieved for $n_{r} \geqslant 160$.

\subsection{Time-marching algorithm}

The integration in time of the incompressible Navier-Stokes equations (2.1) is carried out by a second-order-accurate predictor-corrector fractional-step method (Goda 1979; Raspo et al. 2002). At the intermediate time-step, the velocity components are obtained by solving Helmholtz-type problems. A Poisson problem then yields a correction to the pressure required to enforce divergence-free velocity fields. A CrankNicholson scheme is used for the viscous terms; the advection terms are obtained at the intermediate time-step by extrapolation based on the previous time-steps.

\section{Global flow behaviour and basic velocity fields}

The present section considers the global wake dynamics for moderate Reynolds numbers, $R e \leqslant 350$, and compares its features with those obtained by previous experiments or simulations. The direct numerical simulations carried out here serve two purposes. (1) Validate the numerical code by accurately reproducing the different known flow regimes: steady axisymmetric flow for $R e<R e_{1}$, steady non-axisymmetric flow for $R e_{1}<R e<R e_{2}$ and unsteady flow for $R e>R e_{2}$. (2) Obtain the timeindependent solutions of the Navier-Stokes equations that are used as basic flows for the stability analyses of the next section. These basic flow fields must be computed at all relevant Reynolds numbers, including those for which they are globally unstable: $R e>R e_{1}$ for the axisymmetric and $R e>R e_{2}$ for the non-axisymmetric basic flows. These globally unstable non-axisymmetric basic wakes have been computed for the first time here, by the frequency damping method of Åkervik et al. (2006).

\subsection{Steady axisymmetric flow}

A steady axisymmetric solution

$$
\left.\begin{array}{rl}
\boldsymbol{u} & =\boldsymbol{u}^{A}(r, z ; R e) \\
p & =p^{A}(r, z ; R e)
\end{array}\right\}
$$

of the Navier-Stokes equations (2.1) exists at all Reynolds numbers and can easily be computed via direct numerical simulations. By truncating the Fourier expansions (2.4) at $|\beta| \leqslant N_{h}=0$, the system converges in time towards the steady state (3.1), even for $R e>R e_{1}$ when this basic state is globally unstable to non-axisymmetric perturbations (see 33.2 ).

The structure of the basic axisymmetric wake fields at $R e=100,200$ and 300 is illustrated in figure 1 where isolines of the pressure field $p$ and the azimuthal vorticity $\omega_{\theta} \equiv \partial_{z} u-\partial_{r} w$ are shown. These fields are in excellent agreement with those obtained for $R e<R e_{1}$ by Johnson \& Patel (1999, figures 6 and 7) using a discretization based on spherical coordinates, a spatial grid fitted to the sphere surface and explicitly implementing surface boundary conditions. Thus the ability of the present immersed boundary technique to capture the flow fields with great accuracy is demonstrated.

\subsection{Global instabilities}

At $R e_{1} \simeq 212$, a regular bifurcation occurs (Ghidersa \& Duček 2000) and the axisymmetric flow becomes globally unstable with respect to non-axisymmetric perturbations. Thus, for $R e>R e_{1}$, the wake flow naturally evolves towards a new 
(a)
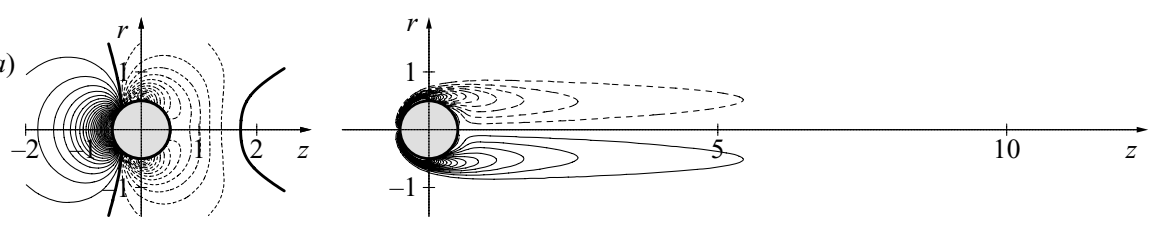

(b)
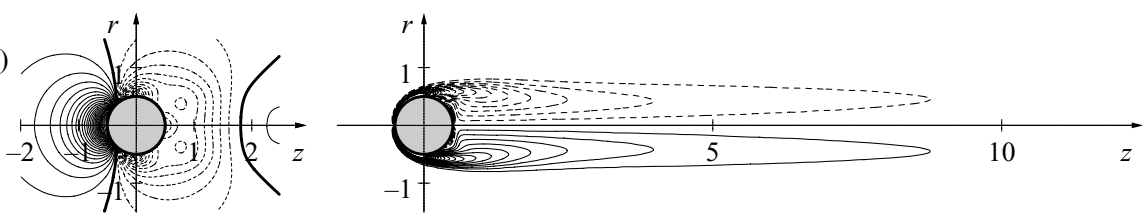

(c)
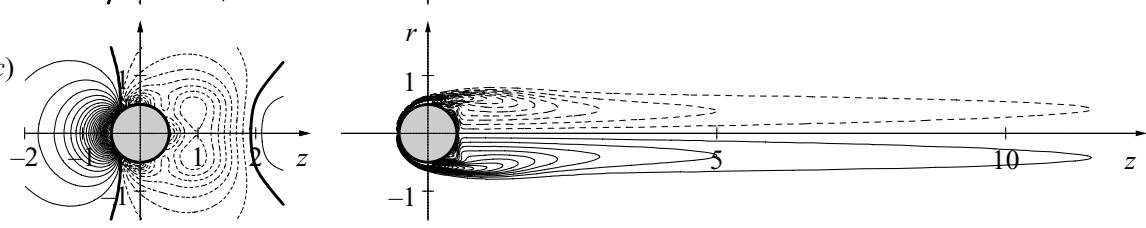

FIGURE 1. Flow structure of steady axisymmetric wake. Isolines of pressure $p$ (left) and azimuthal vorticity $\omega_{\theta}$ (right) at $(a) R e=100,(b) R e=200$ and $(c) R e=300$. Solid (dashed) contours correspond to positive (negative) values. Pressure contours are spaced by 0.04 and vorticity contours by 0.5 .

steady non-axisymmetric state exhibiting a symmetry plane containing the $z$-axis. This steady planar symmetric flow in turn becomes globally unstable at $R e_{2} \simeq 272$, where a Hopf bifurcation leads to periodic vortex shedding.

The breaking of axisymmetry is associated with the appearance of higher azimuthal Fourier modes. Figure 2 shows the temporal evolution of energy contained in the first azimuthal $(\beta=1)$ Fourier component; in these simulations the axisymmetric basic flow solution (3.1) is non-axisymmetrically perturbed at $t=0$. From figure $2(a)$ it is derived that global instability of the axisymmetric state occurs for $210<R e_{1}<220$, while onset of periodic vortex shedding is seen in figure $2(b)$ to occur for $270<$ $R e_{2}<280$. The steady planar symmetric wake obtained with $R e=250$ can be seen in figure 4(a) below, and a snapshot of the vortex shedding regime prevailing at $R e=300$ is shown in figure 3 . The vortex shedding frequency measured for $R e>R e_{2}$ weakly increases with Reynolds number and equals $\omega=0.85$ at $R e=300$, which corresponds to a Strouhal number of $S t=\omega / 2 \pi=0.135$. These results are in good agreement with the usually assumed critical Reynolds numbers of $R e_{1}=212$ and $R e_{2}=272$, as well as the expected Strouhal number (Johnson \& Patel 1999; Ghidersa $\&$ Duček 2000). Since the purpose of the present investigation is not the precise determination of these thresholds, these results are deemed sufficient validation of the code and no further numerical refinement has been pursued.

\subsection{Steady planar symmetric flow}

For $R e>R e_{1}$, there exists a steady non-axisymmetric but planar symmetric solution

$$
\left.\begin{array}{l}
\boldsymbol{u}(r, \theta, z, t) \equiv \boldsymbol{u}^{P}(r, \theta, z ; R e), \\
p(r, \theta, z, t) \equiv p^{P}(r, \theta, z ; R e),
\end{array}\right\}
$$

of the Navier-Stokes equations (2.1). Below, the coordinates are always chosen so that the symmetry plane coincides with the $(x, z)$-plane defined by $\theta=0$. This steady planar symmetric wake flow is globally stable up to $R e_{2}$. A direct numerical simulation 

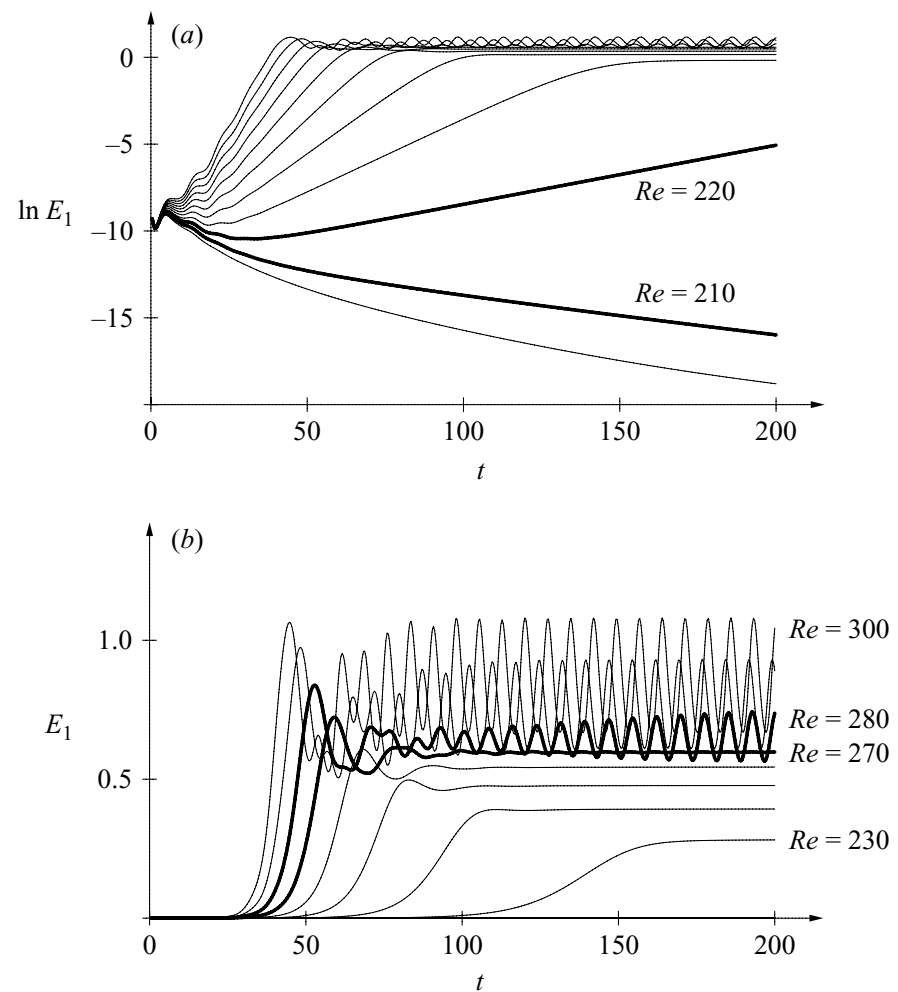

FIGURE 2. Temporal evolution of energy $E_{1}$ (arbitrary units) contained in the first azimuthal Fourier mode for $R e=200,210, \ldots, 300$.

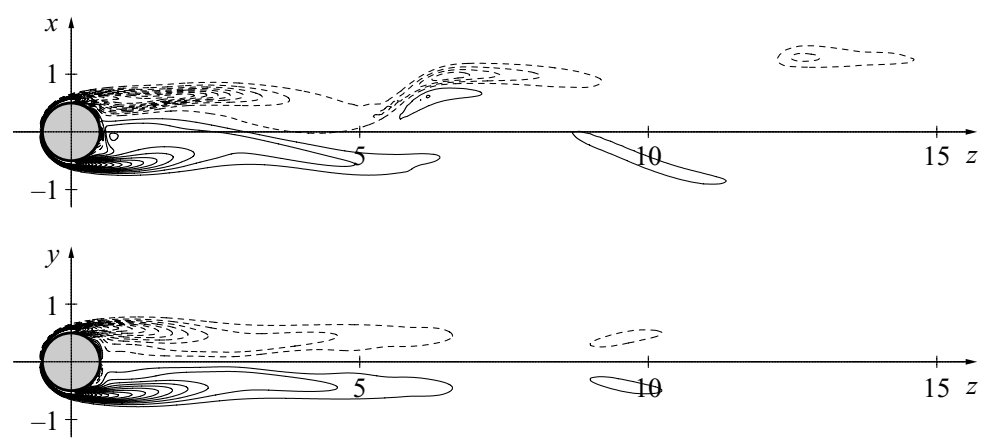

FIGURE 3. Snapshot of periodic vortex shedding regime at $R e=300$. Isolines of azimuthal vorticity $\omega_{\theta}$ in the $(x, z)$-plane (symmetry plane $\left.\theta=0\right)$ and $(y, z)$-plane $(\theta=\pi / 2)$ Solid (dashed) contours correspond to positive (negative) values, spaced by 0.5 .

(with $N_{h}>0$ ) thus evolves in time towards the steady planar symmetric wake for $R e_{1}<R e<R e_{2}$ and towards a time-dependent regime for $R e>R e_{2}$. It should be noted that the onset of periodic vortex shedding does not break the planar symmetry of the underlying unstable basic flow: the time-dependent flow fields (see figure 3) conserve the $(x, z)$-symmetry plane. Thus there is no way to obtain the steady unstable flow solution (3.2) for $R e>R e_{2}$ by imposing an additional symmetry condition in the direct numerical simulations. 
In order to compute the planar symmetric basic flow for $R e>R e_{2}$, a NewtonRaphson search procedure could be numerically implemented that solves for steady solutions of the full system. However, owing to the size of the system of nonlinearly coupled equations, this procedure turned out to be extremely time and memory consuming (even when resorting to approximate iterative methods) and poorly converging.

A much more efficient way to compute the unstable basic flow was to implement the selective frequency damping method of Åkervik et al. (2006). In a nutshell, this frequency damping method enables the computation of steady solutions by adding to the right-hand side of (2.1a) a linear forcing term $\boldsymbol{f}_{d}$ driving the system towards a target solution

$$
\boldsymbol{f}_{d}(r, \theta, z, t)=-\frac{1}{\tau_{d}}(\boldsymbol{u}(r, \theta, z, t)-\overline{\boldsymbol{u}}(r, \theta, z, t)),
$$

where $\tau_{d}$ can be understood as a characteristic damping time. The target solution $\overline{\boldsymbol{u}}$ must be chosen so that it converges towards the unknown steady solution, and this can be achieved by low-pass filtering (with cutoff frequency $1 / \tau_{f}$ ) the already computed velocity fields

$$
\overline{\boldsymbol{u}}(r, \theta, z, t)=\int_{0}^{t} \frac{1}{\tau_{f}} \exp \left(-\frac{t-t^{\prime}}{\tau_{f}}\right) \boldsymbol{u}\left(r, \theta, z, t^{\prime}\right) \mathrm{d} t^{\prime},
$$

or equivalently

$$
\partial_{t} \overline{\boldsymbol{u}}=\frac{1}{\tau_{f}}(\boldsymbol{u}-\overline{\boldsymbol{u}}) .
$$

Time-marching of the target solution governed by (3.5) can be done simultaneously with the simulation of the Navier-Stokes equations (2.1) with $f=f_{b}+f_{d}$ and only requires minimal modifications to the original code. As this coupled system converges towards a steady state, the additional damping term (3.3) vanishes and the resulting time-independent flow fields are exact steady solutions of the original Navier-Stokes equations. In the present investigation, a good compromise between stability of the scheme and fast convergence towards a steady solution was obtained with $\tau_{d}=1$ and $\tau_{f}=10$.

The structure of the steady planar symmetric wake at $R e=200,250$ and 300 is shown in figure 4.

\subsection{Drag, lift and reverse flow}

As a final check, and to conclude this section on the global flow behaviour, the drag and lift forces acting on the sphere as well as the reverse-flow regions in the different wake regimes have also been computed.

As mentioned in $\$ 2.1$, the hydrodynamic forces are here simply obtained by spatial integration of the volume forces (2.3) required by the immersed boundary method. Since these volume forces are designed to maintain the fluid at rest in the domain covered by the sphere, they exactly oppose the hydrodynamic forces due to the surrounding flow. The drag force is the component acting in the $z$-direction. Since the symmetry plane of the wakes is chosen to lie at $\theta=0$, defined as the $(x, z)$-plane, the lift force is obtained by projection onto the $x$-axis, while the lateral force along the $y$-axis vanishes. Drag and lift coefficients $C_{D}$ and $C_{L}$ are shown in figure 5. The thick solid lines correspond to the steady axisymmetric wakes; the thick dashed lines represent the hydrodynamic forces computed for the steady planar symmetric fields. 
(a)
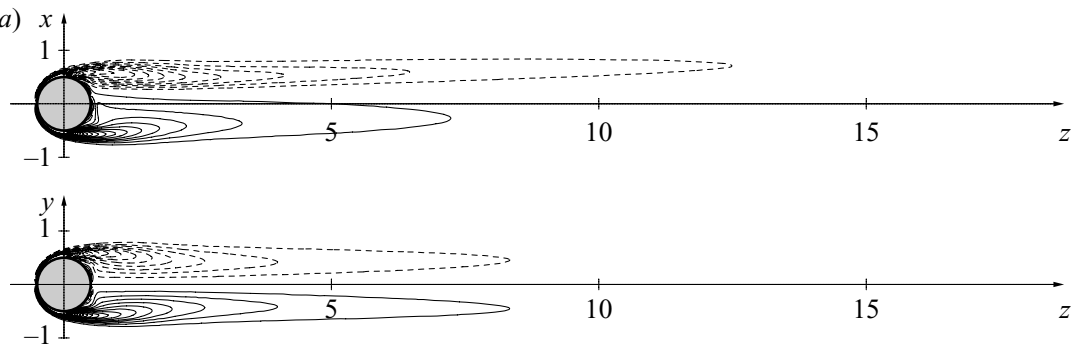

(b)
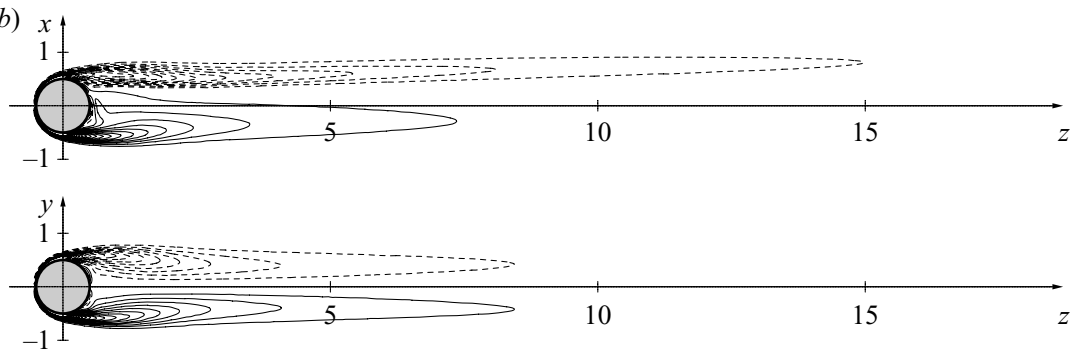

(c)

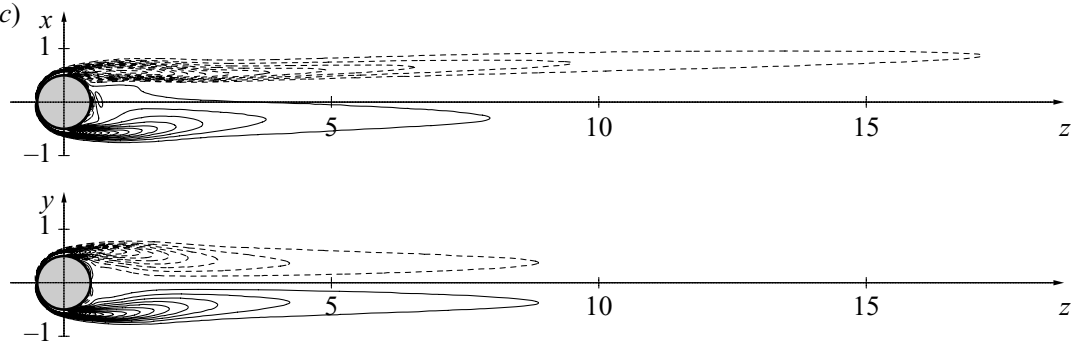

FIGURE 4. Flow structure of a steady planar symmetric wake. Isolines of azimuthal vorticity $\omega_{\theta}$ in $(x, z)$-plane (symmetry plane $\left.\theta=0\right)$ and $(y, z)$-plane $(\theta=\pi / 2)$ at $(a) R e=250,(b)$ $R e=300$ and (c) $R e=350$. Solid (dashed) contours correspond to positive (negative) values, spaced by 0.5 .

Beyond $R e_{2}$, the wake naturally evolves towards a time-dependent state, and the thin lines illustrate the maximum and minimum values in this periodic regime.

Since absolute instability is often associated with the existence of reverse flow, the evolution with Reynolds number of the recirculation region is plotted in figure 6 . For axisymmetric basic wakes, the reverse-flow region terminates at a stagnation point located at $Z_{\star}^{A}$ (solid curve) on the $z$-axis (note that the recirculation length is given by $\left.Z_{\star}^{A}-0.5\right)$. For the steady planar symmetric wakes, two quantities have been computed: the downstream boundary of the reverse flow region $Z_{\star}^{P}$ (dashed curve), defined as the zero-crossing of $\min _{r, \theta} w^{P}(r, \theta, z ; R e)$, and the location $Z_{\star \star}^{P}$ (dotted curve) where the $z$-axis crosses the reverse flow region, i.e. the zero-crossing of $w^{P}(r=0, \theta, z ; R e)$. At $R e=R e_{1}$, the breaking of axisymmetry causes a lateral shift of the reverse-flow region, which entails a drop in $Z_{\star \star}^{P}$. However, reverse flow prevails off the $z$-axis beyond $Z_{\star \star}^{P}$ down to $Z_{\star}^{P}$, which is seen to increase almost linearly with $R e$.

The results shown in figures 5 and 6 are in excellent agreement with the data recently obtained by Bouchet et al. (2006) using a different method; note however that these authors did not compute the globally unstable planar symmetric base flow for $R e>\operatorname{Re}_{2}$. 

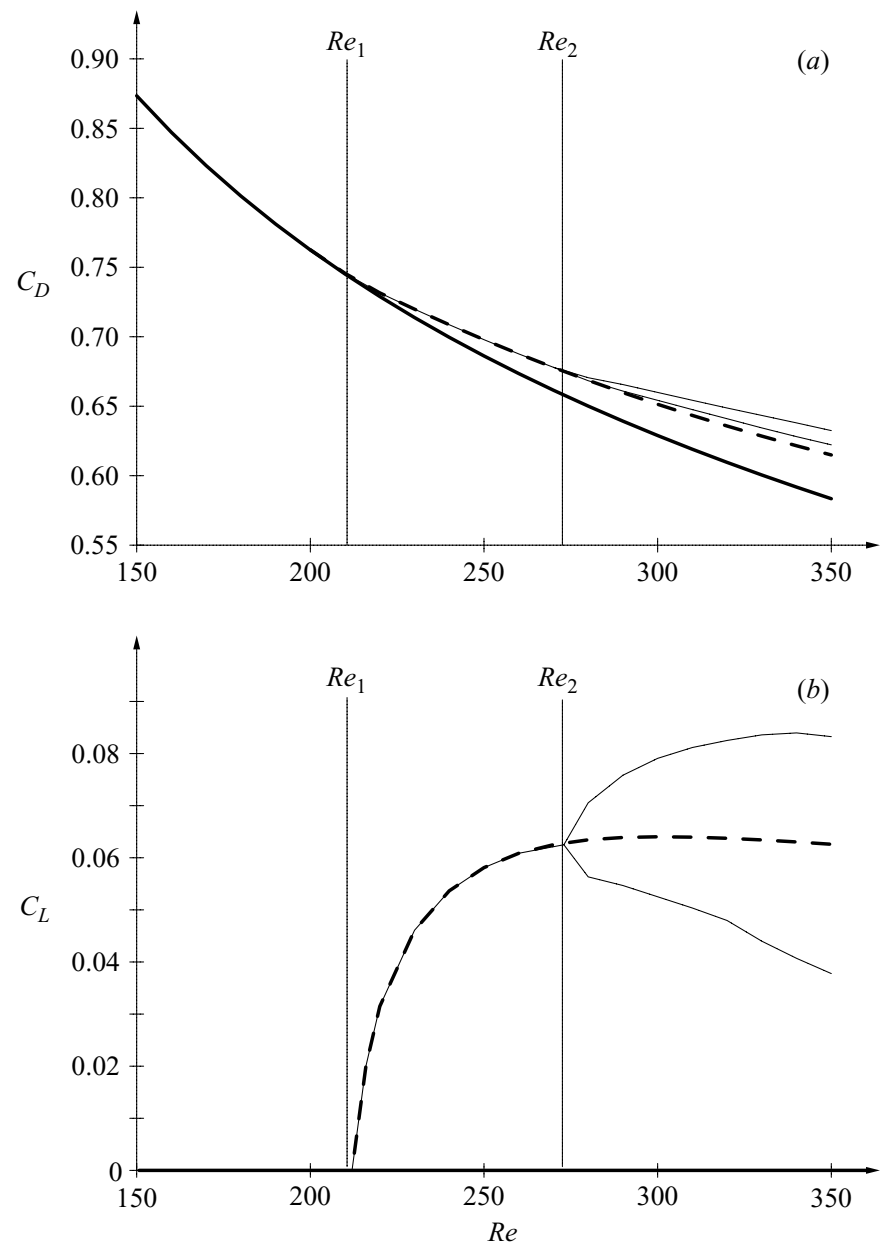

Figure 5. (a) Drag and (b) lift coefficients as a function of the Reynolds number for the different flow regimes: axisymmetric wake (thick solid), steady planar symmetric wake (thick dashed), unsteady planar symmetric wake (two thin lines corresponding to minimum and maximum values).

\section{Local linear stability analyses}

The next two subsections of this study investigate the local stability properties of the previously obtained basic wake flows: both the axisymmetric $\boldsymbol{u}^{A}(r, z ; R e)(3.1)$ and the planar symmetric $\boldsymbol{u}^{P}(r, \theta, z ; R e)$ (3.2) steady solutions of the Navier-Stokes equations. Local properties of these flows, valid at a given axial position $z$, are derived from the velocity profiles prevailing at that station. Such an approach can be justified by a rigorous asymptotic analysis based on the assumption of a slow axial development of the basic flow. The flow around a spherical obstacle is clearly non-parallel in the near-wake region; however, following the successful treatment of the cylinder wake (Pier 2002), we again ignore the non-parallelism of the present flow and study its local stability features. Local properties are then derived by freezing the $z$-coordinate, at say $z=Z$, and studying the equivalent axially parallel shear flows of either axisymmetric or planar symmetric velocity profiles

$$
\boldsymbol{U}^{A}(r ; Z, R e) \equiv \boldsymbol{u}^{A}(r, Z ; R e) \quad \text { or } \quad \boldsymbol{U}^{P}(r, \theta ; Z, R e) \equiv \boldsymbol{u}^{P}(r, \theta, Z ; R e)
$$




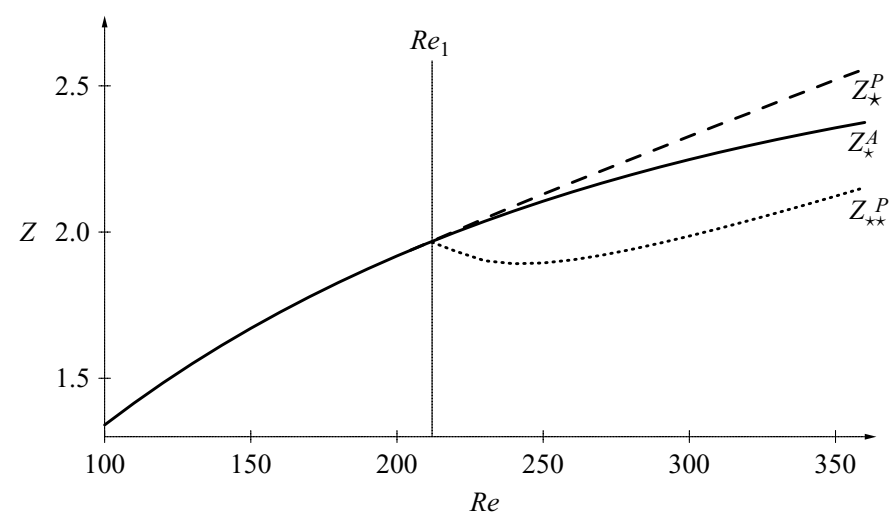

FIGURE 6. Reynolds-number dependence of reverse-flow region. For axisymmetric basic wakes, the recirculation region terminates at $Z_{\star}^{A}$ (solid curve). For planar symmetric basic wakes, it extends down to $Z_{\star}^{P}$ (dashed) and crosses the $z$-axis at $Z_{\star \star}^{P}$ (dotted).

where $Z$ now acts as a parameter rather than a coordinate.

\subsection{Local stability of axisymmetric basic wake}

When studying the local stability properties of the axisymmetric basic wake under the parallel flow assumption, both $z$ and $\theta$ are homogenous directions, and the total flow fields may then be separated into basic and perturbation quantities according to

$$
\left.\begin{array}{l}
\boldsymbol{u}(r, \theta, z, t)=\boldsymbol{U}^{A}(r ; Z, R e)+\boldsymbol{u}^{l}(r ; \alpha, \beta ; Z, R e) \operatorname{expi}(\alpha z+\beta \theta-\omega t), \\
p(r, \theta, z, t)=P^{A}(r ; Z, R e)+p^{l}(r ; \alpha, \beta ; Z, R e) \exp \mathrm{i}(\alpha z+\beta \theta-\omega t) .
\end{array}\right\}
$$

Here the infinitesimally small velocity and pressure disturbances have been written in normal-mode form, where $\alpha$ is a complex axial wavenumber, $\beta$ an integer azimuthal mode number, $\omega$ a complex angular frequency and $\boldsymbol{u}^{l}, p^{l}$ the associated complex velocity and pressure components. After substitution of (4.2) into the Navier-Stokes equations, the linearization in the perturbation quantities yields an eigenvalue problem in the radial direction. By using a Chebyshev spectral method based on the same radial collocation points for which the base flow has been computed, the full $\omega$-spectrum is obtained for each setting of the wavenumbers $\alpha$ and $\beta$ and of the parameters $Z$ and Re. In figure 7, the $\omega$-spectra are shown corresponding to $\alpha=2$ and $|\beta| \leqslant 4$ for the velocity profiles obtained in the axisymmetric wake at $Z=1$ with $R e=300$ (illustrated in figure $1 c$ ). The full spectra are made up of many modes, among which only a few are physically relevant. Note that the numerical method approximates a continuous spectrum at $\omega_{r}=\alpha$, associated with modes oscillating in the free stream. For each $\beta$ the most unstable (or least stable) mode is indicated by a bold dot in figure 7. Identification of these modes yields the local linear dispersion relation

$$
\omega=\Omega^{A}(\alpha, \beta ; Z, R e) .
$$

For axisymmetric base flows, the eigenfunctions $\boldsymbol{u}^{l}(r ; \alpha, \beta)$ and $\boldsymbol{u}^{l}(r ; \alpha,-\beta)$ are associated with the same frequency, i.e. $\Omega^{A}(\alpha, \beta)=\Omega^{A}(\alpha,-\beta)$, and transform into each other under the symmetry operator $\Pi$ (2.5), with respect to the $(\theta=0)$-plane. A planar symmetric eigenmode is thus obtained as the combination $\boldsymbol{u}=\boldsymbol{u}^{l}(r ; \alpha, \beta) \mathrm{e}^{\mathrm{i} \beta \theta}+\boldsymbol{u}^{l}(r ; \alpha,-\beta) \mathrm{e}^{-\mathrm{i} \beta \theta}$, which satisfies $\Pi \boldsymbol{u}=\boldsymbol{u}$, whereas the combination $\boldsymbol{u}=\boldsymbol{u}^{l}(r ; \alpha, \beta) \mathrm{e}^{\mathrm{i} \beta \theta}-\boldsymbol{u}^{l}(r ; \alpha,-\beta) \mathrm{e}^{-\mathrm{i} \beta \theta}$ satisfies $\Pi \boldsymbol{u}=-\boldsymbol{u}$ and thus represents an eigenmode that is antisymmetric with respect to the $(\theta=0)$-plane. For comparison 


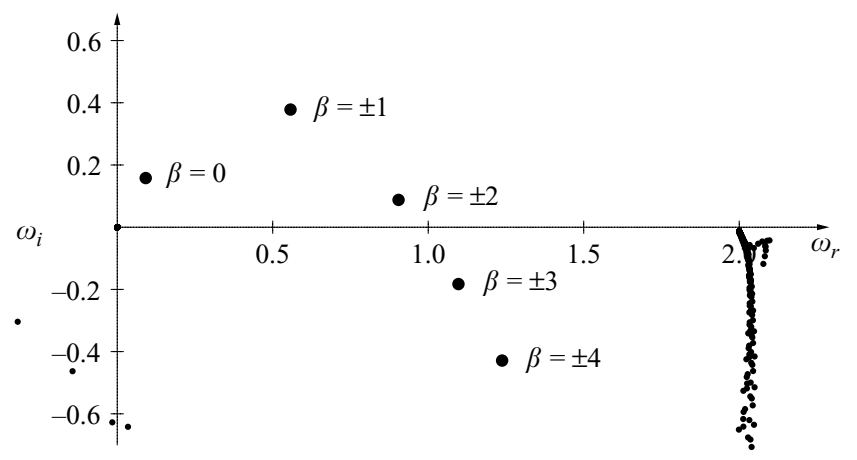

FIGURE 7. Superposition of temporal $\omega$-spectra corresponding to $\alpha=2$ and $\beta=0,1,2,3,4$ for axisymmetric wake flow at $Z=1$ with $R e=300$.
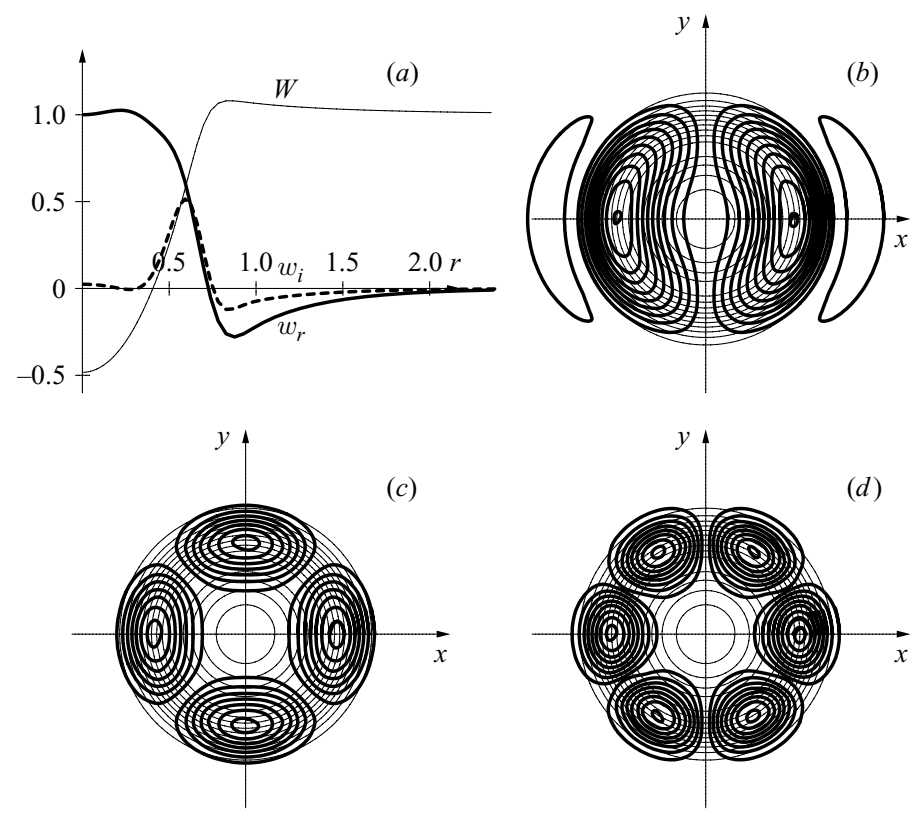

FIGURE 8. Structure of eigenfunctions obtained for axisymmetric wake flow at $Z=1$, $R e=300$ with $\alpha=2$ and (a) $\beta=0,(b) \beta=1,(c) \beta=2,(d) \beta=3$. The axial velocity component $w(r, \theta)$ of the normalized modes (thick curves) is shown together with the axial basic flow component $W(r)$ (thin curves). (a) Radial profile of real $w_{r}$ (thick solid) and imaginary $w_{i}$ (thick dashed) parts of the axisymmetric mode corresponding to $\alpha=2$ and $\beta=0$. ( $(b-d)$ Modulus $|w|$ (thick equispaced isolines) of planar symmetric modes corresponding to $\alpha=2$ and $\beta=1(b), \beta=2(c)$ and $\beta=3(d)$. In $(b-d)$, the basic wake profile $W$ is indicated by thin equispaced isolines.

with eigenmodes pertaining to non-axisymmetric wakes (figure 13), the structure of the symmetric eigenmodes is illustrated in figure 8.

In the context of open shear flows, a crucial feature is the complex absolute frequency defined as the frequency observed at a fixed spatial location in the longtime linear response to an initial impulse. For an axially localized impulse with given azimuthal mode number $\beta$, the local absolute frequency $\omega_{0}^{A}$ and associated absolute axial wavenumber $\alpha_{0}^{A}$ are derived from the linear dispersion relation (4.3) by applying 

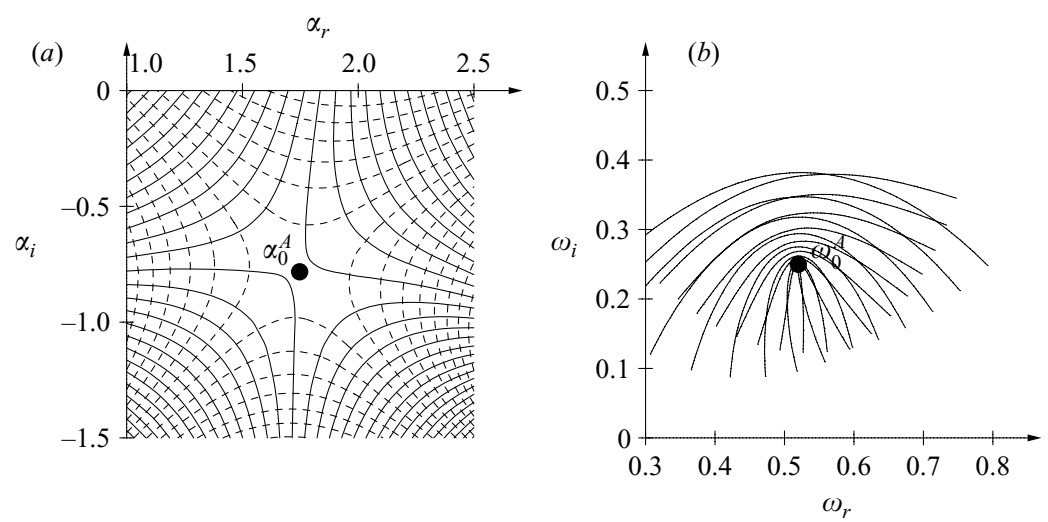

FIGURE 9. Identification of absolute frequency $\omega_{0}$ and wavenumber $\alpha_{0}$ for $\beta=1, Z=1$ in an axisymmetric wake at $R e=300$, by monitoring how the dispersion relation maps the complex $\alpha$-plane onto the complex $\omega$-plane. (a) Isocontours of $\omega_{r}$ (solid) and $\omega_{i}$ (dashed) in the $\alpha$-plane display a saddle point at $\alpha_{0}^{A} \simeq 1.75-0.78 \mathrm{i}$ marked by a solid dot. (b) The temporal curves $\Omega^{A}(\alpha)$ computed for lines of constant $\alpha_{i}$ display a cusp at $\omega_{0}^{A} \simeq 0.52+0.25 \mathrm{i}$ in the $\omega$-plane.

the Briggs (1964) and Bers (1983) pinch-point criterion, equivalent to the vanishing group velocity condition

$$
\omega_{0}^{A}(\beta ; Z, R e)=\Omega^{A}\left(\alpha_{0}^{A}, \beta ; Z, R e\right) \quad \text { and } \quad \frac{\partial \Omega^{A}}{\partial \alpha}\left(\alpha_{0}^{A}, \beta ; Z, R e\right)=0
$$

with the additional requirement that the two spatial $\alpha$-branches that coalesce at the branch-point singularity $\alpha_{0}^{A}$ originate from distinct half- $\alpha$-planes for sufficiently large and positive values of $\omega_{i}$. Pinch points are readily identified by computing the dispersion relation (4.3) on a rectangular grid in the $\alpha$-plane and monitoring how it maps the complex $\alpha$-plane onto the complex $\omega$-plane. Plotting the associated temporal branches in the $\omega$-plane and $\omega_{r}$ - and $\omega_{i}$-isolines in the $\alpha$-plane reveals respectively the characteristic cusp near $\omega_{0}^{A}$ and saddle point near $\alpha_{0}^{A}$. Once a pinch point has thus been approximately located, the exact values of $\omega_{0}^{A}$ and $\alpha_{0}^{A}$ are found by iteratively solving (4.4) with this initial guess. This method is illustrated in figure 9 for $\beta=1$, $Z=1$ and $R e=300$, which yields $\omega_{0}^{A}=0.52+0.25 \mathrm{i}$ and $\alpha_{0}^{A}=1.75-0.78 \mathrm{i}$. The structure of the absolute eigenmodes obtained for azimuthal mode numbers $\beta=0,1$ and 2 at $Z=1$ in an axisymmetric wake with $R e=300$ is given in figure 10 .

The streamwise evolutions of the local absolute growth rate $\omega_{0, i}$ and real absolute frequency $\omega_{0, r}$ are illustrated in figure 11 for $\beta=0,1,2,3$ and axisymmetric basic wakes in the range $100 \leqslant R e \leqslant 350$.

From these plots it is found that the near wake displays absolutely unstable regions when $R e>R e_{a} \simeq 130$ and that the strongest instability always occurs for $|\beta|=1$. Axisymmetric $(\beta=0)$ perturbations are weakly absolutely unstable for $R e>170$ while higher azimuthal modes $(|\beta| \geqslant 2)$ never reach absolute instability at these Reynolds numbers. The discrepancy between onset of local absolute instability at $R e_{a} \simeq 130$ and of global vortex shedding at $R e_{2} \simeq 272$ will be addressed in the concluding section.

\subsection{Local stability of planar symmetric basic wake}

When investigating the local stability features of a planar symmetric basic flow, the local velocity profiles depend on both radial and azimuthal directions, and $z$ is the 

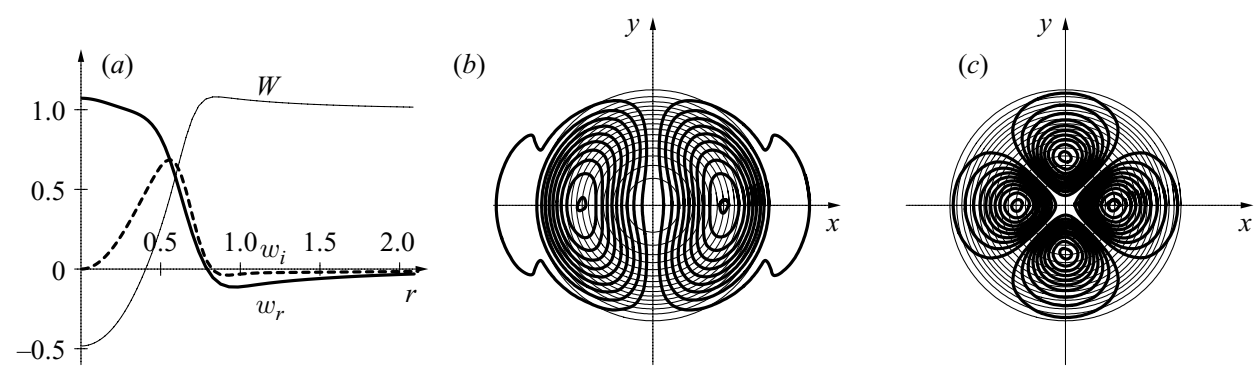

FIGURE 10. Structure of absolute eigenfunctions for axisymmetric wake flows at $Z=1$ and $R e=300$. The axial velocity component $w(r, \theta)$ of the modes (thick curves) is shown together with the axial basic flow component $W(r)$ (thin curves). (a) Axisymmetric mode $\beta=0$, radial profile of real $w_{r}$ (thick solid) and imaginary $w_{i}$ (thick dashed) parts. $(b, c)$ Non-axisymmetric modes $\beta=1$ and $\beta=2$ respectively, equispaced (thick solid) isolines of modulus $|w|$.
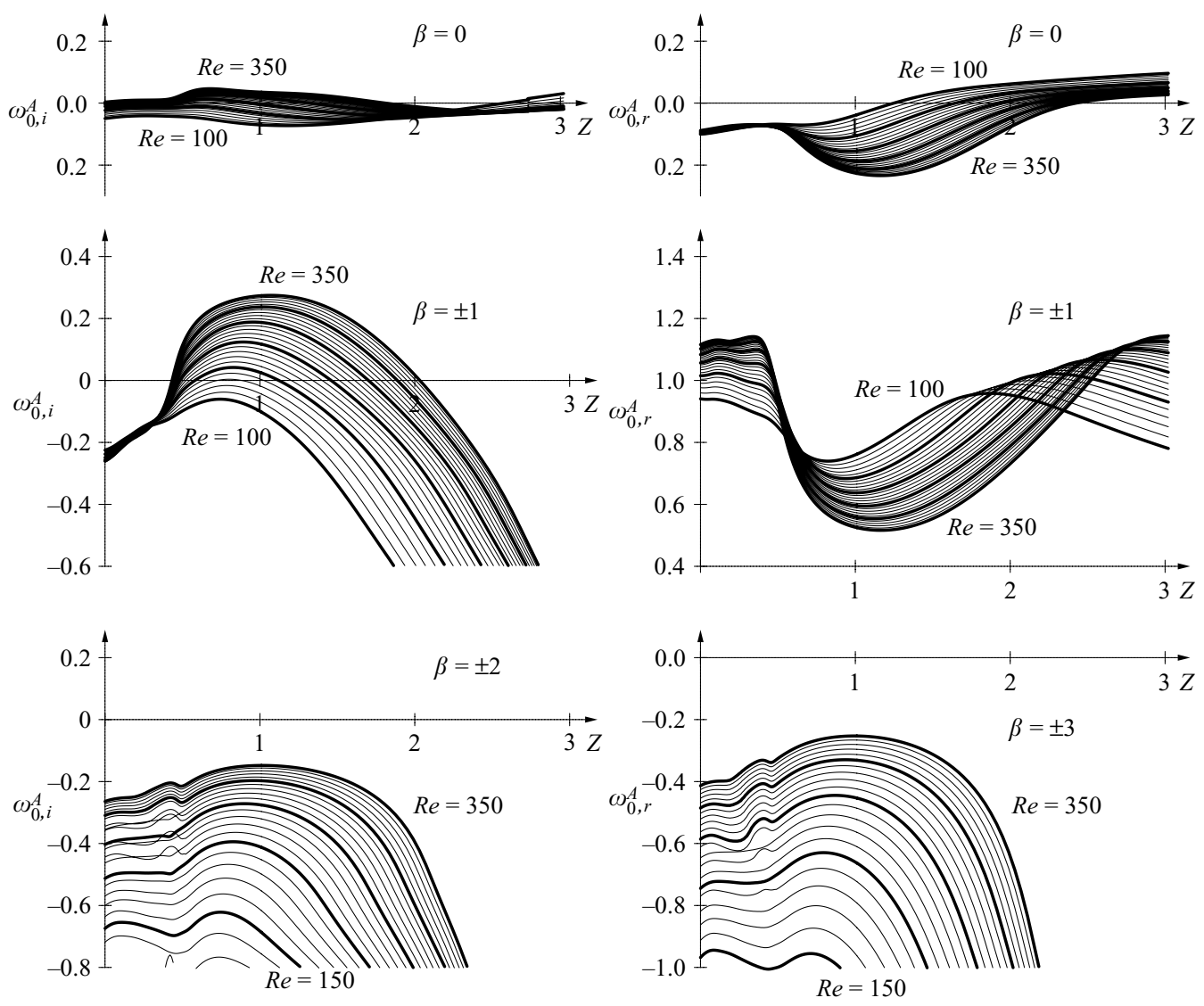

FIGURE 11. Local absolute growth rates $\omega_{0, i}^{A}$ and frequencies $\omega_{0, r}^{A}$ for $|\beta| \leqslant 3$ in axisymmetric basic wake flows at $R e=100,110, \ldots, 350$.

only spatial homogenous coordinate. The total flow fields are then separated into basic and perturbation quantities as

$$
\left.\begin{array}{l}
\boldsymbol{u}(r, \theta, z, t)=\boldsymbol{U}^{P}(r, \theta ; Z, R e)+\boldsymbol{u}^{l}(r, \theta ; \alpha ; Z, R e) \exp \mathrm{i}(\alpha z-\omega t), \\
p(r, \theta, z, t)=P^{P}(r, \theta ; Z, R e)+p^{l}(r, \theta ; \alpha ; Z, \operatorname{Re}) \exp \mathrm{i}(\alpha z-\omega t),
\end{array}\right\}
$$




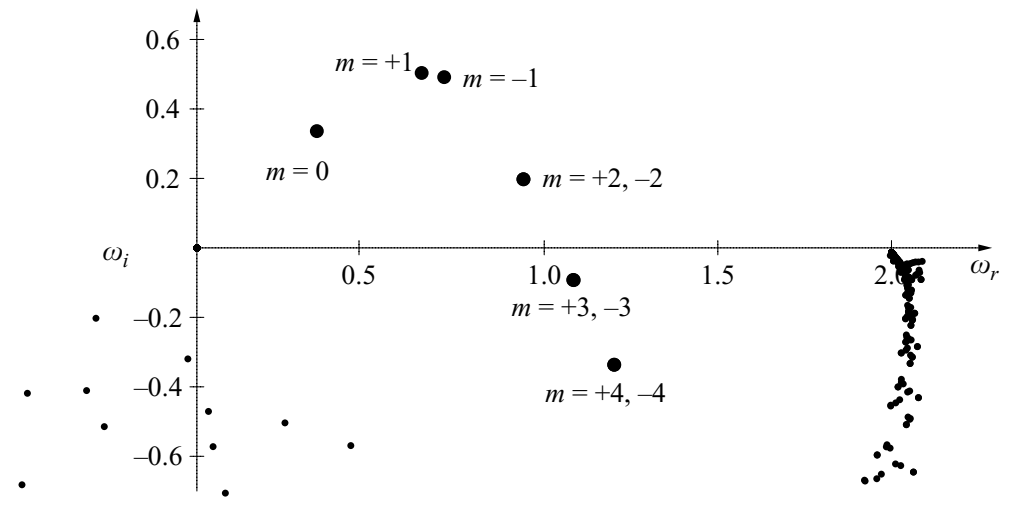

FIGURE 12. Frequency eigenvalues obtained with $\alpha=2$ for non-axisymmetric wake flow at $Z=1$ with $R e=300$.

where $\alpha$ is again a complex axial wavenumber, $\omega$ a complex angular frequency and the eigenmodes $\boldsymbol{u}^{l}$ and $p^{l}$ are now functions of both $r$ and $\theta$ and are governed by the local Navier-Stokes equations linearized about the local planar symmetric wake flow.

For a given Reynolds number above onset of non-axisymmetry $\left(R e>R e_{1}\right)$, computation of the planar symmetric steady wake following the method outlined in $\$ 3.3$ provides the basic flow fields required in (4.5). For these basic flow quantities, the values of their azimuthal Fourier components are known on a set of radial collocation points. Thus the same Fourier-Chebyshev expansion is used for the twodimensional eigenfunctions $\boldsymbol{u}^{l}$ and $p^{l}$ in (4.5), and the associated eigenproblems are then obtained as a large system of linear equations where the different azimuthal harmonics of the eigenfunctions are linearly coupled via the harmonics of the basic flow. The solution of these eigensystems then yields the full $\omega$-spectrum for each setting of the wavenumber $\alpha$ and the parameters $R e$ and $Z$. Since the basic flow is symmetric about the plane $\theta=0$, i.e. invariant under the symmetry operator $\Pi$ (2.5), the associated eigenfunctions are either symmetric $(\Pi \boldsymbol{u}=\boldsymbol{u})$ or antisymmetric $(\Pi \boldsymbol{u}=-\boldsymbol{u})$, and the numerical resolution of the eigenproblems can be speeded up by taking advantage of these symmetry properties. By analogy with two-dimensional jets or wakes, the present symmetric (resp. antisymmetric) modes are also termed varicose (resp. sinuous). Figure 12 shows the frequency spectrum corresponding to $\alpha=2$, computed with the planar symmetric basic wake profile at $Z=1$ for $R e=300$ (illustrated in figure $4 b$ ). By comparison of this spectrum with the corresponding spectra obtained at similar parameter settings for the axisymmetric wake flow (see figure 7), the physically relevant eigenvalues in figure 12 are labelled as $m=0, \pm 1$, $\pm 2, \ldots$ where the values $m \geqslant 0$ denote symmetric or varicose modes while the values $m<0$ denote antisymmetric or sinuous modes. Identification of these modes then yields the local linear dispersion relation governing the planar symmetric wake flows

$$
\omega=\Omega^{P}(\alpha, m ; Z, R e) .
$$

Comparison of figures 7 and 12 reveals that the non-axisymmetric basic flow displays larger growth rates $\omega_{i}$ than the axisymmetric case. Unlike the situation prevailing for axisymmetric velocity profiles where the modes $\pm \beta$ are degenerate, here the non-axisymmetric components of the basic flow lift the degeneracy of the $\pm m$ modes, i.e. $\Omega^{P}(\alpha, m) \neq \Omega^{P}(\alpha,-m)$ for $m \neq 0$. However, the influence of the non-axisymmetric components is relatively weak: only the modes $m= \pm 1$ 

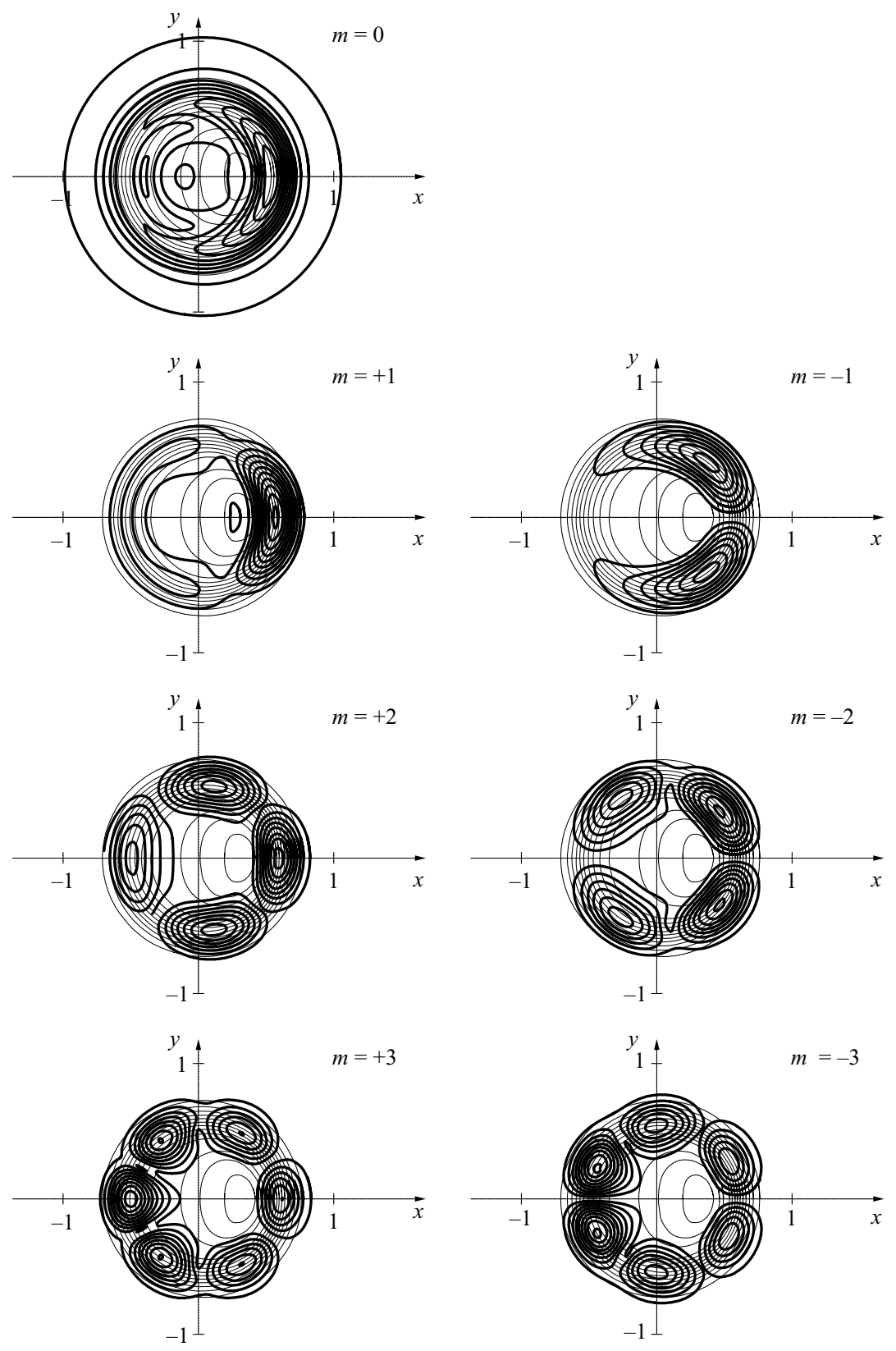

FIGURE 13. Structure of eigenfunctions obtained for planar symmetric wake flow at $Z=1$ and $R e=300$, with $\alpha=2$ and $m=0, \pm 1, \pm 2, \pm 3$. The modulus of the axial velocity component $|w(r, \theta)|$ of the modes (thick equispaced isolines) is shown together with the axial basic flow component $W(r, \theta)$ (thin equispaced isolines).

are clearly distinguished in the frequency plane of figure 12 . The spatial structure of the associated eigenfunctions is illustrated in figure 13 by isolines of $|w|$, the modulus of their complex-valued axial velocity component. Again, only the modes $m= \pm 1$ are clearly differentiated while the higher modes closely resemble their axisymmetric counterparts (see figure 8). Note that plots of $|w|$ are all 

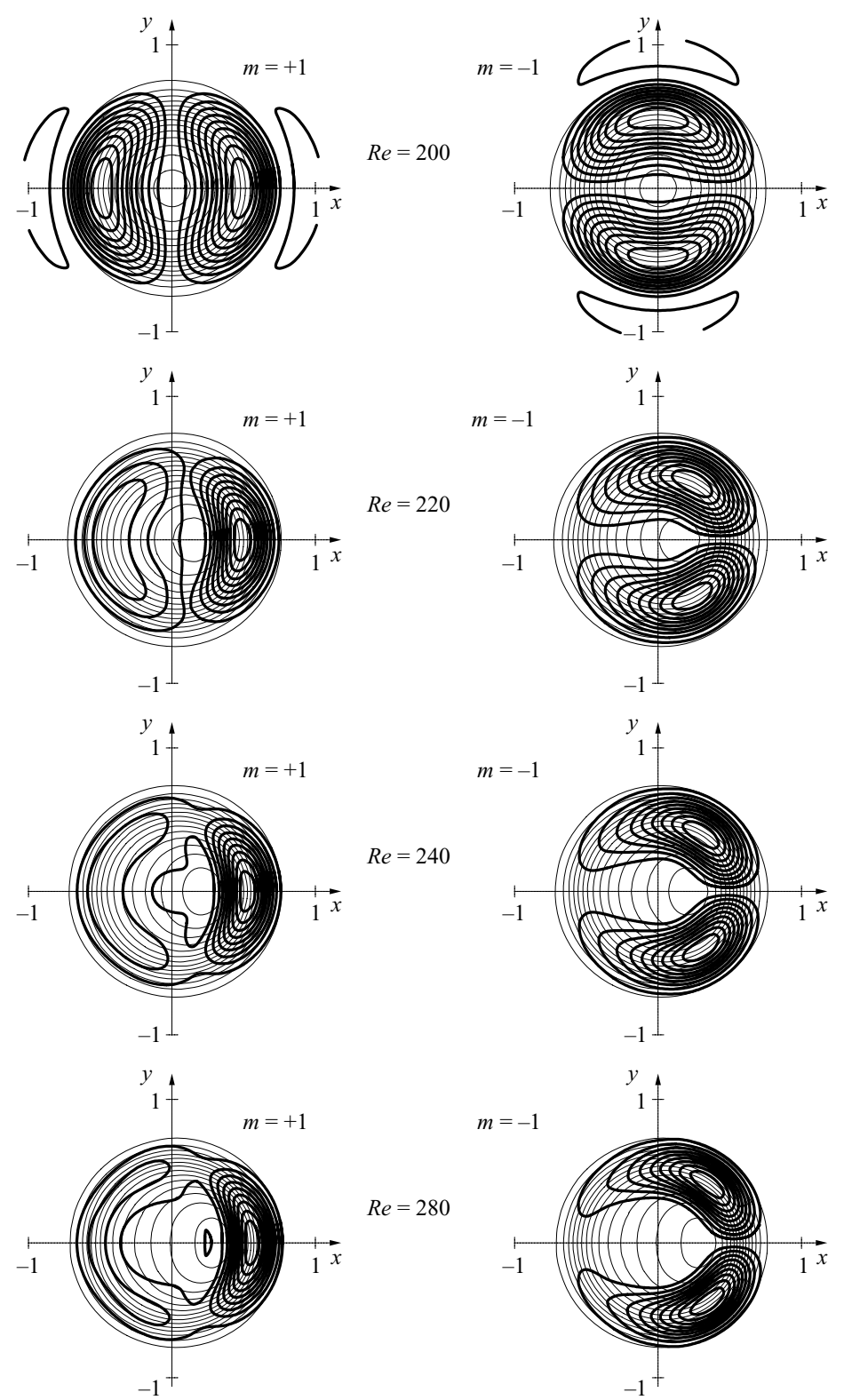

$R e=240$
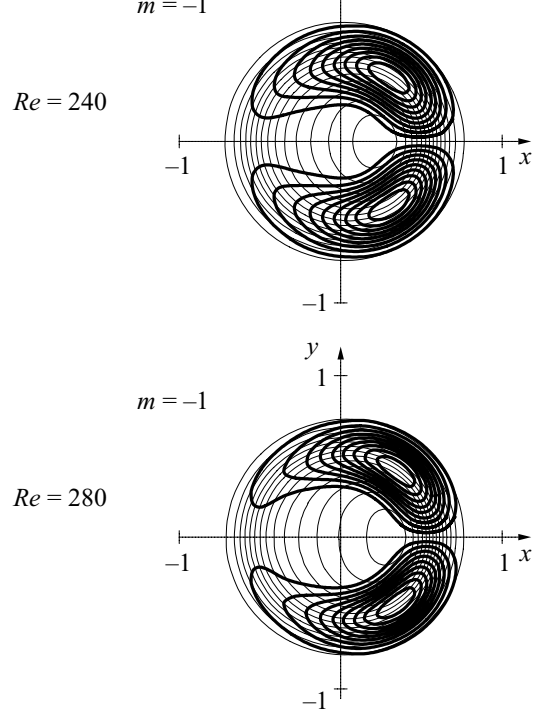

FiguRE 14. Evolution with Reynolds number of planar symmetric $m=+1$ and antisymmetric $m=-1$ eigenfunctions obtained for the basic wake at $Z=1, \alpha=2$ and $R e=200,220$, 240,280 . While the modes are degenerate for the axisymmetric wake prevailing at $R e=200$, they evolve separately for $R e>R e_{1} \simeq 212$ with increasing departure from axisymmetry. The modulus of the axial velocity component $|w(r, \theta)|$ of the modes (thick equispaced isolines) is shown together with the axial basic flow component $W(r, \theta)$ (thin equispaced isolines).

symmetric with respect to the $x$-axis: in this representation antisymmetric eigenmodes are characterized by the vanishing of the $w$ component on the $x$-axis, while planar-symmetric eigenmodes display non-vanishing values of $|w|$ over the $x$-axis. It should be noted also that the unstable eigenfunctions $(|m| \leqslant 2)$ are shifted towards 

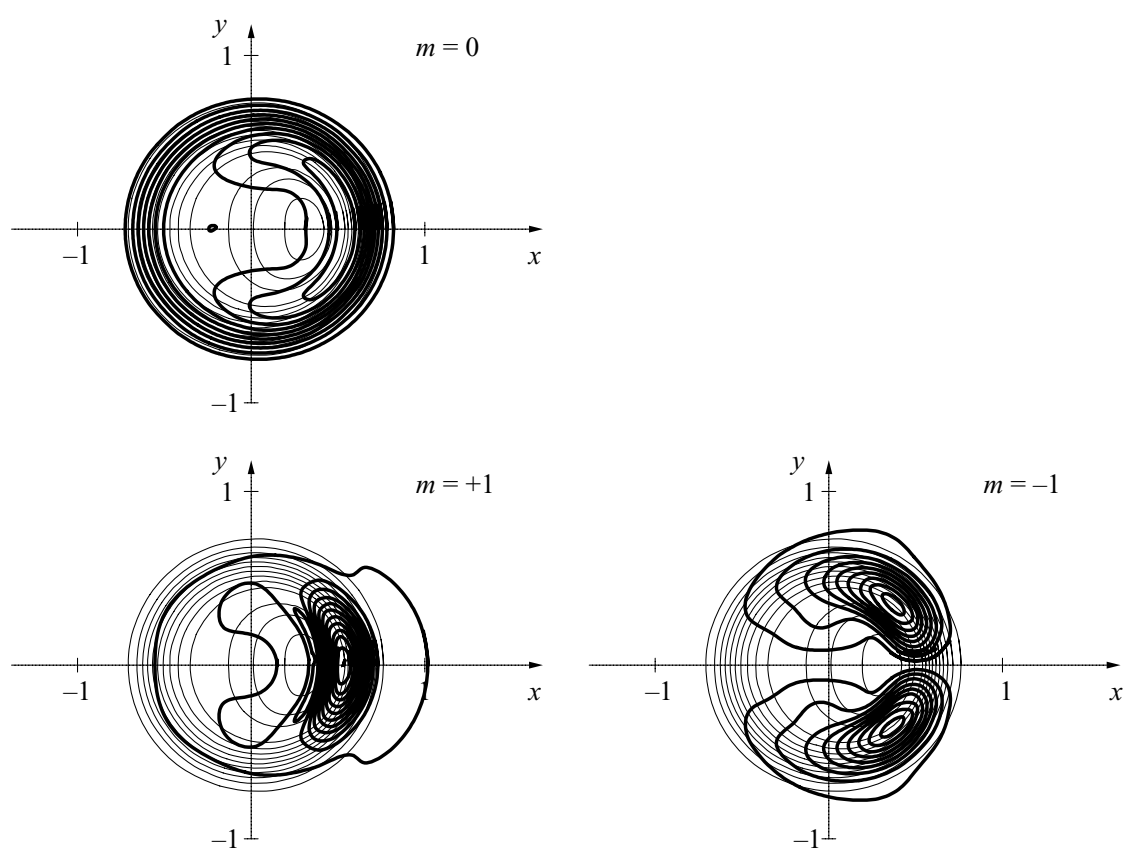

FIGURE 15. Structure of absolute eigenfunctions for planar symmetric wake flow at $Z=1$ and $R e=300$, with $m=0, m=+1$ and $m=-1$. The modulus of the axial velocity component $|w(r, \theta)|$ of the modes (thick equispaced isolines) is shown together with the axial basic flow component $W(r, \theta)$ (thin equispaced isolines).

the regions where the basic wake displays the largest shear, i.e. the neighbourhood of $(x, y)=(0.5,0)$ in these plots.

To further illustrate how the non-axisymmetric modes emerge from their axisymmetric equivalents with increasing Reynolds number, figure 14 plots both planar symmetric $m=+1$ and antisymmetric $m=-1$ eigenmodes obtained with $\alpha=2$ and $Z=1$ for $R e=200,220,240$ and 280. For $R e=200$, below onset of non-axisymmetry, both modes are identical up to a rotation around the $z$-axis. For $R e>R e_{1} \simeq 212$, with increasing departure from axisymmetry, the discrepancy between the $m=+1$ and $m=-1$ modes is seen to increase as they concentrate towards the region of largest shear in the basic flow.

The analysis of absolute instability properties for non-axisymmetric wakes is carried out in a similar fashion as for the axisymmetric case in the previous section; its numerical implementaion, however, is more demanding on computational resources. Application of the Briggs (1964) and Bers (1983) pinch-point criterion with dispersion relation (4.6) yields, for each mode $m$, the local absolute frequency $\omega_{0}^{P}$ and associated absolute axial wavenumber $\alpha_{0}^{P}$ by solving

$$
\omega_{0}^{P}(m ; Z, R e)=\Omega^{P}\left(\alpha_{0}^{P}, m ; Z, R e\right) \quad \text { with } \quad \frac{\partial \Omega^{P}}{\partial \alpha}\left(\alpha_{0}^{P}, m ; Z, R e\right)=0 .
$$

The structure of the absolute eigenfunctions obtained for the modes $m=0,+1$ and -1 for the non-axisymmetric basic wake at $Z=1$ and $R e=300$ is illustrated in figure 15. These modes are associated respectively with the absolute frequencies $\omega_{0}^{P}(m=0) \simeq-0.15+0.03 \mathrm{i}, \omega_{0}^{P}(m=+1) \simeq 0.76+0.41 \mathrm{i}$ and $\omega_{0}^{P}(m=-1) \simeq$ $0.78+0.27 \mathrm{i}$. Comparison with the corresponding absolute modes pertaining to 

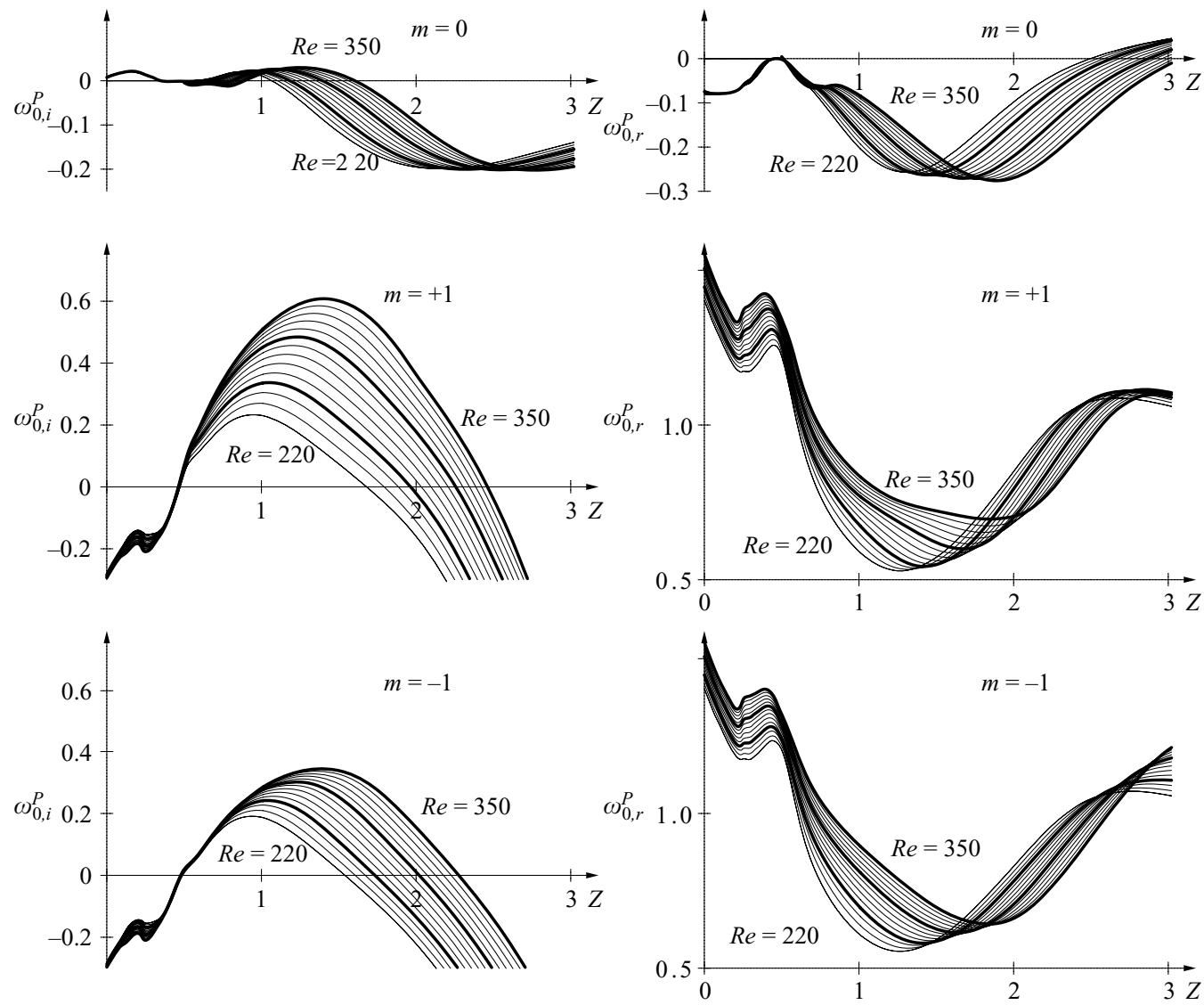

FIGURE 16. Local absolute growth rates $\omega_{0, i}^{P}$ and frequencies $\omega_{0, r}^{P}$ for planar symmetric basic wake flows at $R e=220,230, \ldots, 350$ and $m=0,+1$ and -1 .

axisymmetric wakes (illustrated in figure 10) reveals again that the non-axisymmetric eigenfunctions are concentrated in the regions where the planar symmetric wake displays larger axial shear.

The streamwise evolution of local absolute growth rate $\omega_{0, i}^{P}$ and real absolute frequency $\omega_{0, r}^{P}$ are illustrated in figure 16 for $m=0,+1,-1$ and non-axisymmetric basic wakes in the range $220 \leqslant R e \leqslant 350$.

These results reveal that local absolute instability $\left(\omega_{0, i}^{P}>0\right)$ prevails in the near wake for all Reynolds numbers $R e>R e_{1}$, for which a planar symmetric wake exists. For $R e$ close to $R e_{1}$, the non-axisymmetric components of the basic flow are relatively small, and the symmetric $m=+1$ and antisymmetric $m=-1$ modes display very similar features. With increasing Reynolds number, however, the non-axisymmetric basic wake components strengthen and the $m=+1$ and $m=-1$ modes evolve separately. It is the symmetric $m=+1$ mode that displays the largest absolute instability, while the growth rate of the antisymmetric $m=-1$ increases more weakly with Reynolds number. At $R e=350$, the maximum absolute growth rate of the $m=+1$ mode is in excess of 0.6 , about twice the maximum value reached by the $m=-1$ mode for the same Reynolds number. For the axisymmetric configuration, the $m=0$ modes are at most weakly absolutely unstable while the higher modes $(|m| \geqslant 2$, not shown) do not reach absolute instability. 


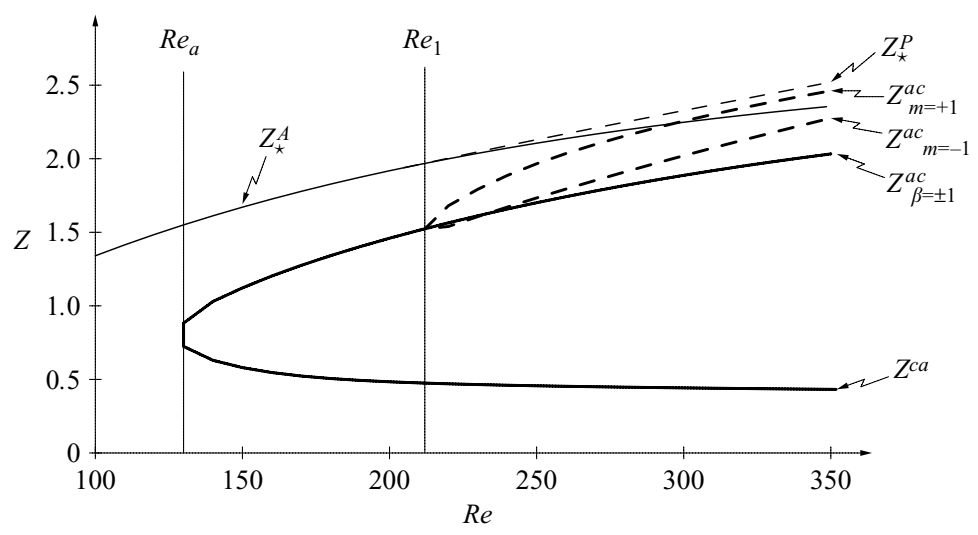

FIGURE 17. Reynolds-number dependence of absolutely unstable (thick curves) and reverse-flow (thin curves from figure 6) regions. For $R e>R e_{a}$, absolute instability prevails over the interval $Z^{c a}<Z<Z^{a c}$, and the different downstream marginal locations correspond to axisymmetric $\left(Z_{\beta= \pm 1}^{a c}\right)$ and planar symmetric $\left(Z_{m=+1}^{a c}\right.$ and $\left.Z_{m=-1}^{a c}\right)$ basic wakes.

\section{Discussion}

Local absolute instability analyses, based on both the axisymmetric and the planar symmetric basic wake flows, have demonstrated the existence of absolutely unstable regions in the near wake. The strength and the spatial extent of the absolute instabilities increase with Reynolds number. The axisymmetric basic wakes display absolute instability for $R e>R e_{a} \simeq 130$; planar symmetric basic wakes exist for $R e>R e_{1} \simeq 212$ and are always absolutely unstable.

For axisymmetric sphere wakes, the largest absolute growth rates are reached for the $\beta= \pm 1$ modes, i.e. the first azimuthal harmonic. This corresponds to the general result (Monkewitz 1988b) that the first helical modes are the most unstable in axisymmetric wake profiles. For planar symmetric basic wakes, the non-axisymmetric basic flow components are found to enhance the instability, and the strongest absolute growth rates are found for the varicose $m=+1$ eigenmodes, which conserve the planar symmetry of the underlying basic flow.

The relationship between absolutely unstable and reverse-flow regions is illustrated in figure 17. For $R e>R e_{a}$, transition from convective to absolute instability occurs at $Z^{c a}$ in the very near wake, and the flow returns to convective instability further downstream at $Z^{a c}$. For axisymmetric wakes, the absolutely unstable region terminates at $Z_{\beta= \pm 1}^{a c}$ where $\omega_{0, i}^{A}(\beta= \pm 1 ; Z, R e)$ changes sign. For planar symmetric wakes, the domains where the $m=+1(m=-1)$ modes display absolute instability are delimited by $Z_{m=+1}^{a c}\left(Z_{m=-1}^{a c}\right)$ defined as zero-crossings of $\omega_{0, i}^{P}(m=+1 ; Z, R e)$ (respectively of $\left.\omega_{0, i}^{P}(m=-1 ; Z, R e)\right)$. As already noted, the varicose $m=+1$ modes developing in the planar symmetric basic wakes display the strongest absolute instabilities: $Z_{m=+1}^{a c}>$ $Z_{m=-1}^{a c}>Z_{\beta= \pm 1}^{a c}$. Comparison of the marginal $Z^{a c}$-curves with the stagnation $Z_{\star}$ curves (from figure 6) shows that reverse flow prevails beyond the absolutely unstable region, both for the axisymmetric and the planar symmetric wakes: $Z_{\star}^{A}>Z_{\beta= \pm 1}^{a c}$ and $Z_{\star}^{P}>Z_{m=+1}^{a c}$. For planar symmetric wakes, however, the curves $Z_{m=+1}^{a c}$ and $Z_{\star}^{P}$ display the same asymptotic trend, which suggests that absolutely unstable and reverse-flow regions exactly overlap in the limit of large Reynolds numbers.

These results confirm the presence of a 'wave-maker' in the near wake of the sphere: the absolutely unstable region sustains fluctuations that develop in situ and 
feed waves into the downstream convectively unstable domain. Moreover, since the dominant instability is a varicose $(m=+1)$ mode, this is in agreement with the observed planar symmetry, rather than antisymmetry, of vortex shedding.

In the context of slowly spatially developing flows, theoretical investigations have shown that the onset of self-sustained nonlinear oscillations coincides with the appearance of local absolute instability (Pier, Huerre \& Chomaz 2001; Chomaz 2005). According to this theory, vortex shedding in the sphere wake should occur as soon as $R e>R e_{a} \simeq 130$ and not only for $R e>R e_{2} \simeq 272$. However, these theoretical analyses rely on the assumption of asymptotically slow streamwise variation, which is clearly not the case in the near wake of a sphere. This strong non-parallelism is thought to be responsible for the discrepancy between onset of absolute instability and onset of global instability. A similar discrepancy prevails for the two-dimensional wake of a circular cylinder, where absolute instability starts at $R e \simeq 25$, while the onset of vortex shedding is at $R e \simeq 47$ (Monkewitz 1988a; Provansal, Mathis \& Boyer 1987; Pier 2002).

Concerning the Strouhal number, the frequency of a self-sustained time-periodic finite-amplitude vortex shedding regime is expected to be governed by the criterion of 'steep' or 'elephant' nonlinear global modes (Pier et al. 1998; Pier \& Huerre 2001). According to this theory, the global frequency equals the real absolute frequency prevailing at the transition location from convective to absolute instability. From the plots of figures 11 and 16, it is seen that transition from convective to absolute instability occurs near the rear boundary of the sphere, for $Z \simeq 0.5$. In this very nearwake region, the associated real absolute frequencies are in the range $0.7<\omega<1.3$, which corresponds to $0.11<S t<0.21$. More specifically, the frequencies prevailing at the convective/absolute transition cover the range $0.7<\omega_{0, r}^{A}<1.0$ for axisymmetric wakes $(130 \leqslant R e \leqslant 350)$ and the range $1.2<\omega_{0, r}^{P}<1.3$ for planar symmetric wakes $(220 \leqslant R e \leqslant 350)$. It would thus appear that the instability properties of the axisymmetric base flows yield frequency predictions closer to the actually observed shedding frequencies $(\omega=0.85$ at $R e=300)$, while the non-axisymmetric base flows somewhat overestimate the shedding frequencies. However, these quantitative differences are thought to be inconclusive since the theoretical frequency selection criterion is based on the assumption of weakly non-uniform systems, while the local absolute frequency displays an important drop in the region $0.5<Z<1$. In view of these strong non-parallel effects in the vicinity of the sphere, the qualitative agreement found with the actually observed frequencies is deemed very reasonable. Note also that for the two-dimensional cylinder wake the global frequencies were similarly found to be systematically below those derived from the local absolute frequencies (Pier 2002). Also for the cylinder wake, Barkley (2006) has recently shown that a linear stability analysis of the mean flow yields better frequency predictions than a nonlinear frequency selection criterion applied to the basic flow. For the three-dimensional wake of a sphere, the relevance of this result and comparisons with different frequency selection criteria (reviewed in Chomaz 2005) are currently being investigated and will be reported in a future publication.

To conclude, it should be emphasized that the motivation of the present study was to completely work out the local absolute instability features of real sphere wakes in order to address the link between these local properties and the fundamental mechanisms dictating the global flow dynamics. While this link is not adequately verified, due, among other factors, to non-parallel effects, the existence of an absolutely unstable pocket has been clearly established. 
The author is indebted to Arie Biesheuvel for triggering interest in the dynamics of the wake of spherical particles and to Jérôme Hœpffner for bringing the frequency damping method to his attention. Stimulating discussions with Marie Rastello and Jean-Louis Marié are gratefully acknowledged.

\section{REFERENCES}

Åkervik, E., Brandt, L., Henningson, D. S., Heepffner, J., Maxen, O. \& Schlatter, P. 2006 Steady solutions of the Navier-Stokes equations by selective frequency damping. Phys. Fluids 18, 068102.

BARKLEY, D. 2006 Linear analysis of the cylinder wake mean flow. Europhys. Lett. 75, 750-756.

BERS, A. 1983 Space-time evolution of plasma instabilities - absolute and convective. In Handbook of plasma physics (ed. M. Rosenbluth \& R. Sagdeev), pp. 451-517. North-Holland.

Bouchet, G., Mebarek, M. \& Dušek, J. 2006 Hydrodynamic forces acting on a rigid fixed sphere in early transitional regimes. Eur. J. Mech. B/Fluids 25, 321-336.

BoyD, J. P. 2001 Chebyshev and Fourier Spectral Methods. Dover.

BriggS, R. J. 1964 Electron-Stream Interaction with Plasmas. MIT Press.

Canuto, C., Hussaini, M. J. \& Quarteroni, A. 1988 Spectral Methods in Fluid Dynamics. Springer.

Chomaz, J.-M. 2005 Global instabilities in spatially developing flows: non-normality and nonlinearity. Annu. Rev. Fluid Mech. 37, 357-392.

Gallaire, F., Ruith, M., Meiburg, E., Chomaz, J.-M. \& Huerre, P. 2006 Spiral vortex breakdown as a global mode. J. Fluid Mech. 549, 71-80.

GHidersa, B. \& DučEK, J. 2000 Breaking of axisymmetry and onset of unsteadiness in the wake of a sphere. J. Fluid Mech. 423, 33-69.

GodA, K. 1979 A multistep technique with implicit difference schemes for calculating two- or three-dimensional cavity flows. J. Comut. Phys. 30, 76-95.

Huerre, P. 2000 Open shear flow instabilities. In Perspectives in Fluid Dynamics (ed. G. K. Batchelor, H. K. Moffatt \& M. G. Worster), pp. 159-229. Cambridge: University Press.

Johnson, T. A. \& Patel, V. C. 1999 Flow past a sphere up to a Reynolds number of 300. J. Fluid Mech. 378, 19-70.

Lesshafft, L., Huerre, P., Sagaut, P. \& Terracol, M. 2006 Nonlinear global modes in hot jets. J. Fluid Mech. 554, 393-409.

MitTAL, R. 1999 Planar symmetry in the unsteady wake of a sphere. AIAA J. 37, 388-390.

Mittal, R. \& Iaccarino, G. 2005 Immersed boundary methods. Annu. Rev. Fluid Mech. 37, 239-261.

Monkewitz, P. A. 1988 a The absolute and convective nature of instability in two-dimensional wakes at low Reynolds numbers. Phys. Fluids 31, 999-1006.

Monkewitz, P. A. 1988 b A note on vortex shedding from axisymmetric bluff bodies. J. Fluid Mech. 192, 561-575.

Nakamura, I. 1976 Steady wake behind a sphere. Phys. Fluids 19, 5-8.

Natarajan, R. \& ACrivos, A. 1993 The instability of the steady flow past spheres and disks. $J$. Fluid Mech. 254, 323-344.

Nichols, J. W., Schmid, P. J. \& Riley, J. J. 2007 Self-sustained oscillations in variable-density round jets. J. Fluid Mech. 582, 341-376.

PIER, B. 2002 On the frequency selection of finite-amplitude vortex shedding in the cylinder wake. J. Fluid Mech. 458, 407-417.

Pier, B. \& Huerre, P. 2001 Nonlinear synchronization in open flows. J. Fluids Struct. 15, 471-480.

Pier, B., Huerre, P. \& Chomaz, J.-M. 2001 Bifurcation to fully nonlinear synchronized structures in slowly varying media. Physica D 148, 49-96.

Pier, B., Huerre, P., Chomaz, J.-M. \& Couairon, A. 1998 Steep nonlinear global modes in spatially developing media. Phys. Fluids 10, 2433-2435.

Provansal, M., Mathis, C. \& Boyer, L. 1987 Bénard-von Kármán instability: transient and forced regimes. J. Fluid Mech. 182, 1-22.

Raspo, I., Hugues, S., Serre, E., Randriamampianina, A. \& Bontoux, P. 2002 A spectral projection method for the simulation of complex three-dimensional rotating flows. Computers Fluids 31, 745-767. 
Roos, F. W. \& Willmarth, W. W. 1971 Some experimental results on sphere and disk drag. AIAA J. 9, 285-291.

Sakamoto, H. \& Haniu, H. 1995 The formation mechanism and shedding frequency of vortices from a sphere in uniform shear flow. J. Fluid Mech. 287, 151-171.

Schouveiler, L. \& Provansal, M. 2002 Self-sustained oscillations in the wake of a sphere. Phys. Fluids 14, 3846-3854.

Sevilla, A. \& Martínez-Bazán, C. 2004 Vortex shedding in high Reynolds number axisymmetric bluff-body wakes: local linear instability and global bleed control. Phys. Fluids 16, 3460-3469.

Tomboulides, A. G. \& Orszag, S. A. 2000 Numerical investigation of transitional and weak turbulent flow past a sphere. J. Fluid Mech. 416, 45-73. 